\title{
Retraction Note to: Inhibition of TMEM16A suppresses growth and induces apoptosis in hepatocellular carcinoma
}

\author{
Chuantao Zhang ${ }^{1} \cdot$ Jianxiang $\mathrm{Liu}^{2} \cdot{\text { Zhiyi } \mathrm{Han}^{3} \cdot \text { Xiang Cui }^{3} \cdot \text { Deti Peng }}^{3} \cdot$ Yufeng Xing ${ }^{3}$
}

Published online: 13 November 2021

๑) The Author(s) under exclusive licence to Japan Society of Clinical Oncology 2021

\section{Retraction Note to: International Journal of Clinical Oncology (2020) 25:1145-1154 https://doi.org/10.1007/s10147-020-01653-6}

The Editor-in-Chief has retracted this article upon request of the corresponding author. Following publication, the authors were unable to replicate the presented results due to the use of the contaminated SMMC7721 cell line in the original experiments. In addition, data duplication has been identified in Figs. 3A, 3C and 5E, raising further concerns regarding the integrity of the data. The authors provided new data to address these issues, but the Editor deemed their response to the concerns insufficient.
All authors agree to this retraction.

Publisher's Note Springer Nature remains neutral with regard to jurisdictional claims in published maps and institutional affiliations.
The original article can be found online at https://doi.org/10.1007/ s10147-020-01653-6.

\section{Yufeng Xing}

yufeng000729@163.com; doctorxingyf@163.com

1 Department of Respiration, Hospital of Chengdu University of Traditional Chinese Medicine, Chengdu 610072, Sichuan, China

2 Key Laboratory of Medicinal Biotechnology, Guilin Medical University, Guilin 541004, Guangxi, China

3 Department of Hepatology, Shenzhen Traditional Chinese Medicine Hospital, NO.1, Fuhua Road, Futian District, Shenzhen 518033, Guangdong, China 